\title{
ON LATTICE ISOMORPHISMS OF INVERSE SEMIGROUPS
}

\author{
PETER R. JONES \\ Department of Mathematics, Statistics and Computer Science, Marquette University, \\ Milwaukee WI 53233, USA \\ e-mail: jones@mscs.mu.edu
}

(Received 13 January 2003; accepted 6 May, 2003)

\begin{abstract}
An $\mathcal{L}$-isomorphism between inverse semigroups $S$ and $T$ is an isomorphism between their lattices $\mathcal{L}(S)$ and $\mathcal{L}(T)$ of inverse subsemigroups. The author and others have shown that if $S$ is aperiodic - has no nontrivial subgroups then any such isomorphism $\Phi$ induces a bijection $\phi$ between $S$ and $T$. We first characterize the bijections that arise in this way and go on to prove that under relatively weak 'archimedean' hypotheses, if $\phi$ restricts to an isomorphism on the semilattice of idempotents of $S$, then it must be an isomorphism on $S$ itself, thus generating a result of Goberstein. The hypothesis on the restriction to idempotents is satisfied in many applications. We go on to prove theorems similar to the above for the class of completely semisimple inverse semigroups.
\end{abstract}

2000 Mathematics Subject Classification. Primary 20M18, secondary 08A30.

Over the past quarter-century, several authors have investigated the extent to which an inverse semigroup $S$ is determined by its lattice $\mathcal{L}(S)$ of inverse subsemigroups (see the survey [8] and the monograph [12]): given an $\mathcal{L}$-isomorphism, that is, an isomorphism $\Phi: \mathcal{L}(S) \rightarrow \mathcal{L}(T)$ for some inverse semigroup $T$, how are $S$ and $T$ related? It is easily seen that since $\Phi$ restricts to an $\mathcal{L}$-isomorphism between their respective semilattices of idempotents, $E_{S}$ and $E_{T}$, it induces a bijection $\phi_{E}$ between them. Following the lead of Goberstein [4] we focus here on the situation where $\phi_{E}$ is an isomorphism (see below for a rationale for this simplification).

It has long been known that $\phi_{E}$ extends to a bijection $\phi: E_{S} \cup N_{S} \rightarrow E_{T} \cup N_{T}$, where $N_{S}$ denotes the set of elements that belong to no subgroup of $S$. In the aperiodic (or 'combinatorial') case where, by definition, all subgroups are trivial, $\phi$ is then a bijection between $S$ and $T$. In turn, $\phi$ induces $\Phi$ in the obvious way. In this note we first characterize the bijections so obtained, in Theorem 2.3, and then in Theorem 4.3 find a general sufficient condition in order that this bijection should be an isomorphism, improving on some results of Goberstein [4].

Since groups are not generally determined by their subgroup lattices, proving lattice determinability of nonaperiodic inverse semigroups must involve either some assumptions on the lattice determinability of the subgroups or some structural 'tyingin' of the subgroups into the overall form of the semigroup. It was shown by Ershova (see [12]) that as long as each nonaperiodic $\mathcal{D}$-class contains at least two idempotents (essentially a statement about the principal factors) then the partial bijection $\phi$ can be extended to a bijection $\theta$ between $S$ and $T$. If $S$ is also completely semisimple, $\theta$ again induces $\Phi$. Again, we characterize the bijections that can arise in this way, in Theorem 2.5. We go on to show in Theorem 4.5 that, under similar hypotheses to the aperiodic case, $\theta$ is again an isomorphism. However, we shall see that assuming that $\Phi$ 
induces isomorphisms on both the semilattice of idempotents and the principal factors does not suffice to induce an isomorphism on the semigroup itself, without the extra hypothesis of Theorem 4.5 .

The assumption that $\phi_{E}$ be an isomorphism is satisfied in many circumstances. For instance this occurs whenever $S$ is simple or is $E$-unitary with a greatest $\mathcal{J}$-class which is nontrivial [7]. It also arises from external conditions. For instance, Goberstein showed (loc. cit.) that "most" isomorphisms between the inverse semigroups of partial automorphisms of two inverse semigroups induce an $\mathcal{L}$-isomorphism of this type. In [2], [3], K. H. Cheong and the author studied $\mathcal{C} o$-isomorphisms between inverse semigroups - isomorphisms between their lattices of convex inverse subsemigroups (which are not generally sublattices of the lattices of all inverse subsemigroups) and reduced their study to those that induce an isomorphism between the respective semilattices of idempotents. In the completely semisimple case, they showed that $\mathrm{Co}_{0}$ isomorphisms with this property are equivalent to $\mathcal{L}$-isomorphisms of the same type. This equivalence is not true in general.

The two theorems on the bijections that induce $\mathcal{L}$-isomorphisms were inspired by Cheong's initial work on the corresponding problem for $\mathcal{C} o$-isomorphisms.

1. Preliminaries. The lattice $\mathcal{L}(S)$ of inverse subsemigroups of an inverse semigroup $S$ has as its zero the empty inverse subsemigroup. If $U, V \in \mathcal{L}(S)$, their join is denoted $U \vee V$ and is the same as their join as subsemigroups. If $A \subseteq S$, we denote by $\langle A\rangle$ the inverse subsemigroup that it generates. The idempotents of $S$ form a semilattice, denoted $E_{S}$. An inverse subsemigroup of $S$ is full if it contains $E_{S}$. The filter $\left[E_{S}, S\right]$ in $\mathcal{L}(S)$ is denoted $\mathcal{L} \mathcal{F}(S)$. Note that for a group $G, \mathcal{L} \mathcal{F}(G)$ coincides with the subgroup lattice but the empty inverse subsemigroup must be adjoined to obtain $\mathcal{L}(G)$.

The natural partial order on an inverse semigroup $S$ is defined by $a \leq b$ if $a=a a^{-1} b$; various useful equivalent properties may be found in [11], along with such basic semigroup concepts as Green's relations, ideals and principal factors. The notation $a \| b$ means that $a$ and $b$ are incomparable with respect to that order. An inverse subsemigroup $U$ is convex if $a \in S, u, v \in U$ and $u \leq a \leq v$ imply that $a \in U$. The convex inverse subsemigroups form a lattice $\mathcal{C} o(S)$ which is not, in general, a sublattice of $\mathcal{L}(S)$. See [2], [3] for a comprehensive study of that lattice, with applications of the results contained herein.

An inverse semigroup is aperiodic, or combinatorial, if all of its subgroups are trivial; $S$ is completely semisimple if each principal factor is completely 0 -simple or is a group (equivalently, $S$ contains no bicyclic subsemigroup - see below for the definition of the latter); $S$ is E-unitary if whenever $a \geq e \in E_{S}$ then $a \in E_{S}$.

We review the basics of $\mathcal{L}$-isomorphisms of inverse semigroups. For surveys see [8] and [12]. Let $\Phi: \mathcal{L}(S) \rightarrow \mathcal{L}(T)$ be an isomorphism. Since $E_{S} \Phi=E_{T}, \Phi$ restricts to an isomorphism $\mathcal{L}\left(E_{S}\right) \rightarrow \mathcal{L}\left(E_{T}\right)$. It also follows that $\Phi$ restricts to an isomorphism $\mathcal{L} \mathcal{F}(S) \rightarrow \mathcal{L} \mathcal{F}(T)$.

Any $\mathcal{L}$-isomorphism $\Phi$ between semilattices $E$ and $F$ induces a bijection $\phi: E \rightarrow F$ by the rule $\langle e \phi\rangle=\langle e\rangle \Phi, e \in E$, and is in turn induced by $\phi$, in the sense that $A \Phi=A \phi$ for every subsemilattice $A$. The bijection $\phi$ is characterized by the weak isomorphism property: if $e, f \in E$ then $e \| f$ if and only if $e \phi \| f \phi$, in which case $(e f) \phi=e \phi f \phi$.

If $\Phi$ is an $\mathcal{L}$-isomorphism between inverse semigroups $S$ and $T$, we shall denote the weak isomorphism between $E_{S}$ and $E_{T}$ by $\phi_{E}$. Note that the class of groups is closed under $\mathcal{L}$-isomorphisms, since groups are characterized among inverse semigroups by 
the property that their semilattices of idempotents are trivial, and $\phi_{E}$ is bijective. Hence if $e \in E_{S}$ then $\Phi$ restricts to an $\mathcal{L}$-isomorphism between the maximal subgroups $H_{e}$ and $H_{e \phi_{E}}$. In particular, if $S$ is aperiodic, so is $T$.

Let $N_{S}$ denote the set of elements of $S$ that do not belong to a subgroup. For each $a \in N_{S}$, by [7] there is a unique element $b$ of $T$ such that $\langle a\rangle \Phi=\langle b\rangle,\left(a a^{-1}\right) \phi_{E}=$ $b b^{-1}$ and $\left(a^{-1} a\right) \phi_{E}=b^{-1} b$. Rephrasing this statement, setting $\left\{b, b^{-1}\right\}=\left\{a \phi_{N}, a^{-1} \phi_{N}\right\}$ yields choice functions $\phi_{N}: N_{S} \rightarrow N_{T}$ that are bijections (since the same process may be applied to $\left.\Phi^{-1}\right)$, and satisfy $a^{-1} \phi_{N}=\left(a \phi_{N}\right)^{-1},\left\{a a^{-1}, a^{-1} a\right\} \phi_{N}=\left\{a \phi_{N}\left(a \phi_{N}\right)^{-1}\right.$, $\left.\left(a \phi_{N}\right)^{-1} a \phi_{N}\right\}$ and $\langle a\rangle \Phi=\left\langle a \phi_{N}\right\rangle$ for every $a \in N_{S}$. Since $\phi_{E}$ also satisfies the above properties, its union with any $\phi_{N}$ yields a bijection $\phi: E_{S} \cup N_{S} \rightarrow E_{T} \cup N_{T}$ with the same properties. The uniqueness of $b$ implies that any bijection that induces $\Phi$ is of this type. Note that by always choosing $a \phi_{N}$ to be $b$ itself, the partial bijection $\phi$ will then uniquely preserve $\mathcal{L}$ and $\mathcal{R}$. We follow [12] in terming this the 'base partial bijection'. While it will be convenient to make that assumption when necessary, we shall not always do so. We summarize the above discussion.

RESULT 1.1. Let $\Phi$ be an $\mathcal{L}$-isomorphism between inverse semigroups $S$ and $T$. There is a bijection $\phi: E_{S} \cup N_{S} \rightarrow E_{T} \cup N_{T}$, unique up to possible interchange of a $\phi$ and $a^{-1} \phi$ for each $a \in S$, such that $\phi$ restricts to a weak isomorphism from $E_{S}$ to $E_{T},\langle a\rangle \Phi=\langle a \phi\rangle$ and $\left\{a a^{-1}, a^{-1} a\right\} \phi=\left\{a \phi(a \phi)^{-1},(a \phi)^{-1} a \phi\right\}$ for every $a \in E_{S} \cup N_{S}$.

There is a unique choice of $\phi$ that preserves $\mathcal{L}$ and $\mathcal{R}$, namely the base partial bijection.

If $S$ is aperiodic, $\phi$ becomes a bijection of $S$ upon $T$ that induces $\Phi$, the properties of which we now summarize. The final statement below is a compilation of results of Ershova and the author that may be found in [12, Section 42].

RESUlT 1.2. Let $S$ be an aperiodic inverse semigroup. Any $\mathcal{L}$-isomorphism $\Phi$ from $S$ to a (necessarily aperiodic) inverse semigroup $T$ is induced by a bijection $\phi: S \rightarrow T$, unique up to possible interchange of $a \phi$ and $a^{-1} \phi$ for each $a \in S$, such that $\phi$ restricts to a weak isomorphism from $E_{S}$ to $E_{T}$ and $\left\{a a^{-1}, a^{-1} a\right\} \phi=\left\{a \phi(a \phi)^{-1},(a \phi)^{-1} a \phi\right\}$ for every $a \in S$. There is a unique choice of $\phi$ that preserves $\mathcal{L}$ and $\mathcal{R}$, namely the base bijection. That choice of $\phi$ restricts to an isomorphism $\langle a\rangle \rightarrow\langle a \phi\rangle$ for each $a \in S$.

We now turn to the nonaperiodic situation, keeping in mind the remarks in the introduction. Ershova showed (see [12, Section 43.7]) that the base partial bijection $\phi: E_{S} \cup N_{S} \rightarrow E_{T} \cup N_{T}$ may be extended to a bijection $\theta: S \rightarrow T$ as long as each nonaperiodic $\mathcal{D}$-class contains at least two idempotents, as follows. For each idempotent $e$ in such a $\mathcal{D}$-class, choose an element $r_{e}$ of $N_{S} \cap R_{e}$. Each $a \in H_{e}$ may be expressed uniquely in the form $a=r_{e} s^{-1}$ for some $s \in H_{r_{e}}$; put $a \theta=r_{e} \phi(s \phi)^{-1}$. (Define $\theta=\phi$ on $N_{S}$.) It follows from the construction that since $\phi$ preserves $\mathcal{L}$ and $\mathcal{R}$, so does $\theta$. It was also shown that if $\Phi$ is induced by an isomorphism $\kappa: S \rightarrow T$, then $\kappa=\theta$. Again following [12], when $\theta$ is defined it is termed the base bijection associated with $\Phi$.

We conclude these preliminaries with some further background on inverse semigroups.

According to [11, Theorem IX.3.11], each monogenic inverse semigroup is defined by exactly one of the following relations, where $k, l$ are positive integers: (i) $a^{k}=a^{-1} a^{k+1}$; (ii) $a^{k} a^{-1}=a^{-1} a^{k}$; (iii) $a^{k}=a^{k+l}$; (iv) $a=a$. Each has a type associated with it: those in (i) are of type $\left(k, \infty^{+}\right)$and possess a bicyclic kernel (least ideal); those in (ii) are of type $(k, \infty)$ and have an infinite cyclic group kernel; those in (iii) are of type $(k, l)$ and have a finite cyclic group kernel; that in (iv) is free. In the first three cases, if $k=1$ then the semigroup itself is bicyclic, infinite cyclic or finite cyclic, respectively. If 
$k \geq 2$, then it is an extension of its kernel by the quotient of the free monogenic inverse semigroup modulo the ideal generated by $a^{k}$ (the quotient being a semigroup of type $(k, 1))$.

A semigroup is group bound, or an epigroup, if some power of each element belongs to a subgroup. From the classification above, it can be seen that a monogenic inverse semigroup is group bound if and only if its semilattice of idempotents is finite. In general, therefore, an inverse semigroup is group bound if and only if it contains no free monogenic nor bicyclic inverse subsemigroup. All periodic inverse semigroups are group bound.

We review from [11, page 47] the construction of retract ideal extensions. Let $B$ be an inverse semigroup and $A$ an inverse semigroup wih zero. Put $A^{*}=A-\{0\}$. Suppose $\psi: A^{*} \rightarrow B$ is a partial homomorphism, that is, $a \psi a^{\prime} \psi=\left(a a^{\prime}\right) \psi$ whenever $a, a^{\prime}, a a^{\prime} \in A^{*}$. Then the set $S=A^{*} \cup B$ becomes an inverse semigroup under the operation that extends that in $B$; extends the partial operation in $A^{*}$; is given by $a a^{\prime}=a \psi a^{\prime} \psi$ when $a, a^{\prime} \in A^{*}$ but $a a^{\prime}=0$ in $A$; and by $a b=a \psi b$ when $a \in A^{*}, b \in B$, and similarly for $b a$. It is the retract (and ideal) extension of $B$ by $A$ defined by $\psi$. (Taking the union of $\psi$ with the identity map on $B$ yields a retraction of $S$.)

\section{Characterizing the induced bijection.}

LEMMA 2.1. Let $S$ and $T$ be inverse semigroups and suppose $\phi: S \rightarrow T$ is a bijection that induces an isomorphism $\mathcal{L} \mathcal{F}(S) \rightarrow \mathcal{L} \mathcal{F}(T)$. If $a, b \in S, b \notin E_{S}$ and $b<a$ then $b \phi \leq(a \phi)^{n}$ for some nonzero integer $n$.

Proof. If $b<a$ then $b=b b^{-1} a \in E_{S} \vee\langle a\rangle$, the full inverse subsemigroup of $S$ generated by $a$, so $b \phi \in E_{T} \vee\langle a \phi\rangle$. Since $E_{S} \phi=E_{T}$, if $b \notin E_{S}$ then $b \phi \notin E_{T}$. Thus $b \phi$ can be expressed as the product of an idempotent and a nonzero power of $a \phi$, that is, $b \phi \leq(a \phi)^{n}$ for some nonzero integer $n$.

Following the terminology of [9], a product $a b$ in an inverse semigroup is restricted if $a^{-1} a=b b^{-1}$, in which case $a \mathcal{R} a b \mathcal{L} b$.

The following lemma is well known, in that it may also be deduced from the standard constructions of the multiplication on an inverse semigroup by way of the restricted products and the partial order (for example, see [9]).

LEMMA 2.2. Let $S$ be an inverse semigroup. A subset $A$ of $S$ is an inverse subsemigroup if and only if $(a)$ it is closed under restricted products and inverses, $(b) E_{A}$ is a subsemilattice of $E_{S}$ and $(c)$ if $b \in S, b b^{-1} \in A$ and $b<a \in A$, then $b \in A$.

Proof. Necessity of (a) and (b) is obvious; that of (c) follows from the equation $b=b b^{-1} a$. To prove sufficiency, let $a, b \in A$. Then $a b=\left(a b b^{-1}\right)\left(a^{-1} a b\right)$, where the product on the right hand side is restricted. It suffices, then, to show that $a^{-1} a b \in A$ (that $a b b^{-1} \in A$ follows dually - observe that condition (c) is actually self dual, since the natural partial order respects inverses). Now $a^{-1} a b \leq b \in A$ and $\left(a^{-1} a b\right)\left(a^{-1} a b\right)^{-1}=$ $\left(a^{-1} a\right)\left(b b^{-1}\right)$ so it remains by (c) to show that $\left(a^{-1} a\right)\left(b b^{-1}\right) \in A$. The product $a^{-1} a$ is restricted, so $a^{-1} a \in A$, and similarly for $b b^{-1}$. Then (b) implies that the product belongs to $A$.

THEOREM 2.3. Let $S$ and $T$ be aperiodic inverse semigroups. Any $\mathcal{L}$-isomorphism $\Phi$ from $S$ to $T$ is induced by a unique bijection $\phi: S \rightarrow T$ satisfying

(1) $\phi$ restricts to a weak isomorphism from $E_{S}$ to $E_{T}$; 
(2) $\phi$ preserves $\mathcal{L}$ and $\mathcal{R}$;

(3) if $b<a$ in $S, b \notin E_{S}$, then $b \phi \leq(a \phi)^{n}$ for some integer $n$, and similarly for $\phi^{-1}$.

Conversely, any bijection $\phi: S \rightarrow T$ that satisfies (1), (2) and (3) induces an $\mathcal{L}$ isomorphism from $S$ to $T$.

The condition (3) can be replaced by (3'): $\phi$ induces an isomorphism of $\mathcal{L} \mathcal{F}(S)$ on $\mathcal{L} \mathcal{F}(T)$.

Proof. The existence of the unique bijection $\phi: S \rightarrow T$ satisfying (1) and (2) was shown in $\S 1$. Clearly, since $\phi$ induces $\Phi$, it induces the restriction of $\Phi$ to $\mathcal{L} \mathcal{F}(S)$. Thus (3) follows from Lemma 2.1.

To prove the converse, assume (1), (2) and (3) hold. (The final statement is immediate from Lemma 2.1.) It follows that $(a \phi)^{-1}=a^{-1} \phi$ for all $a \in S$. It is easily seen that $\phi^{-1}$ also satisfies (1) and (2). Hence, by symmetry it suffices to show that if $A \in \mathcal{L}(S)$ then $A \phi \in \mathcal{L}(T)$. We apply Lemma 2.2. Clearly $A \phi$ is closed under inverses. Since weak isomorphisms are precisely the bijections between semilattices that induce $\mathcal{L}$-isomorphisms between them, $E_{A \phi}$ is a subsemilattice of $E_{T}$. Let $a, b \in A$, with $(a \phi)^{-1} a \phi=b \phi(b \phi)^{-1}$, so that $a \phi \mathcal{R} a \phi b \phi \mathcal{L} b \phi$. Then since $\phi$ preserves $\mathcal{L}$ and $\mathcal{R}, a^{-1} a=b b^{-1}$, so $a \mathcal{R} a b \mathcal{L} b$ and therefore $a \phi \mathcal{R}(a b) \phi \mathcal{L} b \phi$. By aperiodicity, $a \phi b \phi=(a b) \phi \in A \phi$. So $A \phi$ is closed under restricted products. Finally, suppose $b \in S, a \in A$, with $b \phi(b \phi)^{-1} \in A \phi$ and $b \phi<a \phi$. Since $\phi$ preserves $\mathcal{R}$, $b \phi(b \phi)^{-1}=\left(b b^{-1}\right) \phi$, so $b b^{-1} \in A$ and by (3), applied to $\phi^{-1}, b \leq a^{n}$ for some integer $n$. But $a^{n} \in A$ so, by Lemma 2.2, $b \in A$ and $b \phi \in A \phi$, as required.

By the observations preceding Result 1.1, the condition (2) can be replaced by $\left(2^{\prime}\right)\left\{a a^{-1}, a^{-1} a\right\} \phi=\left\{a \phi(a \phi)^{-1},(a \phi)^{-1} a \phi\right\}$ for every $a \in S$ as long as uniqueness of $\phi$ is replaced by uniqueness up to possible interchange of $a \phi$ and $a^{-1} \phi$ for each $a \in S$. This result then characterizes the bijections that can induce an $\mathcal{L}$-isomorphism between aperiodic inverse semigroups.

We now turn to the nonaperiodic situation and characterize the bijections that induce $\mathcal{L}$-isomorphisms. In general, of course, an $\mathcal{L}$-isomorphism between groups is not induced by a bijection. To obtain a complete analogue to the previous theorem we shall need to assume that, in the semigroups under consideration, every nonaperiodic $\mathcal{D}$-class contains at least two idempotents, so that an $\mathcal{L}$-isomorphism $\Phi$ from $S$ to $T$ induces the base bijection $\theta: S \rightarrow T$ defined in Section 1 .

It seems that little is known of the properties of $\theta$ outside the completely semisimple situation. However, it was shown in [12, Lemma 44.5] that if $S$ is a Brandt semigroup that is not a group with zero, then $\theta$ induces $\Phi$ and the question was raised when this occurs in general. We may extend the lemma to all completely semisimple inverse semigroups of the appropriate type, without difficulty. It is known [7] that the class of such semigroups is closed under $\mathcal{L}$-isomorphisms.

LEMMA 2.4. Let $S$ be a completely semisimple inverse semigroup in which each nonaperiodic $\mathcal{D}$-class contains at least two idempotents. If $\Phi$ is an $\mathcal{L}$-isomorphism from $S$ to an inverse semigroup $T$ then it is induced by the base bijection $\theta: S \rightarrow T$.

Proof. Let $a \in S$. If $a \in E_{S} \cup N_{S}$ then $\langle a\rangle \Phi=\langle a \phi\rangle=\langle a \theta\rangle$. So suppose $a \in H_{e}, a \notin E_{S}$, $e \in E_{S}$. Put $r=r_{e}, a=r s^{-1}$ and $a \theta=r \phi(s \phi)^{-1} \in H_{e \phi}$. Following the same argument as in the lemma cited above, based on the fact that the principal factor associated with $e$ is a Brandt semigroup (see [12, Exercise 7b, page 126]), $\langle a\rangle=\langle r, s\rangle \cap H_{e}$ and $\langle a \theta\rangle=\langle r \phi, s \phi\rangle \cap H_{e \phi}=\langle r, s\rangle \Phi \cap H_{e} \Phi=\left(\langle r, s\rangle \cap H_{e}\right) \Phi=\langle a\rangle \Phi$. Hence $\theta$ induces $\Phi$. 
THEOREM 2.5. Let $S$ and $T$ be any completely semisimple inverse semigroups. An $\mathcal{L}$ and $\mathcal{R}$-preserving bijection $\theta: S \rightarrow T$ induces an $\mathcal{L}$-isomorphism $\Phi$ between $S$ and $T$ if and only if

(1) $\theta$ restricts to a weak isomorphism from $E_{S}$ to $E_{T}$;

(2) if $b<a$ in $S, b \notin E_{S}$, then $b \theta \leq(a \theta)^{n}$ for some integer $n$, and similarly for $\theta^{-1}$;

(3) for each $e \in E_{S}, \theta$ induces an $\mathcal{L}$-isomorphism between the subgroups $H_{e}$ and $H_{e \theta}$;

(4) if $a b$ is a restricted product in $S$ and $a, b \in N_{S}$ then (i) if $a b \in N_{S}$ then $(a b) \theta=a \theta b \theta$ and (ii) otherwise (ab) $\theta$ and $a \theta b \theta$ generate the same subgroup of $T$.

If each nonaperiodic $\mathcal{D}$-class of $S$ and $T$ contains at least two idempotents, then any $\mathcal{L}$-isomorphism between them is induced by a unique $\mathcal{L}$-and $\mathcal{R}$-preserving bijection, namely the base bijection $\theta$.

Proof. The last statement follows from Lemma 2.4. Otherwise, we follow the general outline of the proof of the previous theorem. Necessity of (1) has already been noted. That of (2) follows from the same argument as in the previous theorem. Since $\Phi$ restricts to an $\mathcal{L}$-isomorphism between $H_{e}$ and $H_{e \phi_{E}}=H_{e \theta}$, (3) is immediate. To prove (4) we shall need to use complete semisimplicity in a similar manner to that of the proof of Lemma 2.4 .

If $x \in N_{S}$ then $\langle x\rangle \cap D_{x}$ consists of the four distinct elements $x, x^{-1}, x x^{-1}, x^{-1} x$ (since its principal factor is a Brandt semigroup). Similarly, if $a b$ is a restricted product in $S$ and $a, b, a b$ all belong to $N_{S}$, then since $a b \in R_{a} \cap L_{b},\langle a, b\rangle \cap D_{a}=(\langle a\rangle \cup\langle b\rangle \cup$ $\langle a b\rangle) \cap D_{a}$. (See [12, Exercise 7(a), page 126].) Since $\theta$ preserves $\mathcal{L}$ and $\mathcal{R}$, the product $a \theta b \theta$ is also restricted in $T$ and $a \theta, b \theta, a \theta b \theta \in N_{T}$. Hence $\langle a \theta, b \theta\rangle \cap D_{a \theta}=(\langle a \theta\rangle \cup$ $\langle b \theta\rangle \cup\langle a \theta b \theta\rangle) \cap D_{a \theta}$. Since $\langle a, b\rangle \theta=\langle a \theta, b \theta\rangle$, and since $(a b) \theta \in R_{a \theta} \cap L_{b \theta}$ then, in view of the description of $\langle x\rangle \cap D_{x}$ given above, this element belongs to $\langle a \theta b \theta\rangle$ and is $\mathcal{H}$ related to $a \theta b \theta$. Applying that same description, we obtain $(a b) \theta=a \theta b \theta$.

Suppose $a b$ is a restricted product where $a, b \in N_{S}$ but $a b \notin N_{S}$. In that case $a \in H_{b^{-1}}$ and $a b \in H_{f}$, where $f=a a^{-1}=b^{-1} b$. Since $\theta$ induces an $\mathcal{L}$-isomorphism between $H_{f}$ and $H_{f \theta},\langle(a b) \theta\rangle=\langle a b\rangle \theta$. Because the principal factor containing $a$ is a Brandt semigroup, $\langle a b\rangle=\langle a, b\rangle \cap H_{f}$ (see [12, Exercise 7(b), page 126]). Because $\theta$ induces $\Phi,(\langle a, b\rangle \cap$ $\left.H_{f}\right) \theta=\langle a \theta, b \theta\rangle \cap H_{f \theta}$. Finally, since $\theta$ preserves $\mathcal{L}$ and $\mathcal{R},\langle a \theta, b \theta\rangle \cap H_{f \theta}=\langle a \theta b \theta\rangle$, similarly.

To prove the converse, assume (1) - (4) hold. It is again easily seen that $\theta^{-1}$ also satisfies these conditions. Hence it suffices to prove that if $A \in \mathcal{L}(S)$ then $A \theta \in \mathcal{L}(T)$. That $A \theta$ satisfies (b) and (c) of Lemma 2.2 proceeds as in the proof of the previous theorem. It therefore remains to show that it is closed under inverses and restricted products. To show the former property, let $a \in A$. If $a \notin N_{S}$, then $(a \theta)^{-1} \in\langle a \theta\rangle=$ $\langle a\rangle \theta \subseteq A \theta$, using (3). If $a \in N_{S}$ then $a^{-1} \in N_{S}$ and the product $a a^{-1}$ is restricted. By (1)(ii), $\left\langle a \theta a^{-1} \theta\right\rangle=\left\langle\left(a a^{-1}\right) \theta\right\rangle=\left\{\left(a a^{-1}\right) \theta\right\}=\left\{a \theta(a \theta)^{-1}\right\}$, whence since $\theta$ preserves $\mathcal{L}$ and $\mathcal{R},(a \theta)^{-1}=a^{-1} \theta$.

Now suppose $a \theta b \theta$ is a restricted product in $A \theta$. Since $\theta$ and $\theta^{-1}$ preserve $\mathcal{L}$ and $\mathcal{R}$, $a b$ is a restricted product in $A$. There are various cases to consider. Suppose $a, b \in N_{S}$. That $a \theta b \theta \in A \theta$ is immediate from the two cases of (4). If neither $a$ nor $b$ belongs to $N_{S}$ then since $a^{-1} a=b b^{-1}$, both lie in a subgroup $H_{e}, e \in E_{S}$, say, and the requisite conclusion follows from (3).

If $a \notin N_{S}, a \in H_{e}$, say, and $b \in N_{S}$ then $a \mathcal{R} b$ and $a b \mathcal{H} b$, so that the product $(a b) b^{-1}$ is restricted, with $a b, b^{-1} \in N_{S}$. Similarly, $a \theta \in H_{e \theta}, b \theta \in N_{T}$ and $a \theta b \theta \mathcal{H} b \theta$. Let $c=(a \theta b \theta) \theta^{-1} \mathcal{H} b$. Then the product $c b^{-1}$ in $S$ is restricted, with $c b^{-1} \in H_{e}$. By (4)(ii), $\left\langle\left(c b^{-1}\right) \theta\right\rangle=\left\langle c \theta b^{-1} \theta\right\rangle$ in $H_{e \theta}$. Now since $b \in N_{S}, b^{-1} \theta=(b \theta)^{-1}$, as proved above. Thus 
$\left\langle c \theta b^{-1} \theta\right\rangle=\left\langle a \theta b \theta(b \theta)^{-1}\right\rangle=\langle a \theta\rangle$. But from (3), $\langle a \theta\rangle=\langle a\rangle \theta$ and $\left\langle\left(c b^{-1}\right) \theta\right\rangle=\left\langle c b^{-1}\right\rangle \theta$, so $\langle a\rangle=\left\langle c b^{-1}\right\rangle$. Therefore $c b^{-1}=a^{n}$ for some integer $n$ and $c=a^{n} b \in A$. Hence $a \theta b \theta=c \theta \in A \theta$. The case where $a \in N_{S}, b \notin N_{S}$ is dual.

Similar comments to those following the previous theorem apply, regarding the condition that $\theta$ be $\mathcal{L}$ - and $\mathcal{R}$-preserving.

Remark: the second paragraph of the proof is essentially that of the corresponding case of Lemma 4.4 below, and of the result of Ershova cited there (see [12, 44.6.3(a)]).

3. Archimedean properties. In this section we treat the appropriate hypotheses under which we shall eventually prove that every $\mathcal{L}$-isomorphism induces an isomorphism. Goberstein [4] introduced the notion of a 'shortly connected' inverse semigroup and proved (Theorem 5) that any $\mathcal{L}$-isomorphism between such semigroups, with the property that the weak isomorphism $\phi_{E}$ is actually an isomorphism, is induced by a unique isomorphism. He also introduced a slightly stronger property, 'shortly linked', which is more natural and more easily verified. (He showed in the sequel [5] that these two properties are distinct.) For that reason, we prefer to work with generalizations of the latter property.

An inverse semigroup $S$ is shortly linked if for any idempotent $e$ of $S$ and any element $a$ of $S$ such that $e<a a^{-1}$, the set $F_{e, a}=\left\{f \in E_{\langle a\rangle}: e<f \leq a a^{-1}\right\}$ is finite. (Goberstein actually introduced this property in a different form, then showed it to be equivalent to the above in [4, Proposition 3].) Many inverse semigroups turn out to be shortly linked in one of two ways: by virtue of the property that they contain only finitely many idempotents above any given one; or by virtue of being group bound.

Proposition 3.1. (1) An inverse semigroup is shortly linked if and only if no idempotent is strictly below infinitely many idempotents of any monogenic inverse subsemigroup;

(2) every group bound, and hence every periodic and every finite inverse semigroup, is shortly linked;

(3) every free inverse semigroup and monogenic inverse semigroup is shortly linked.

Proof. (1) Let $S$ be an inverse semigroup and $e \in E_{S}, a \in S$. Since every idempotent of $\langle a\rangle$ is below either $a a^{-1}$ or $a^{-1} a,\left\{f \in E_{\langle a\rangle}: e<f\right\}=F_{e, a} \cup F_{e, a^{-1}}$, from which the stated equivalence is clear.

(2) A group bound inverse semigroup is characterized by the property that each of its monogenic inverse subsemigroups has finite semilattice of idempotents.

(3) By [10] every free inverse semigroup $S$ is "finite $\mathcal{J}$-above", that is, for any $x \in S$ there are only finitely many elements $y$ such that $S x S \subseteq S y S$. Hence no idempotent can be strictly below infinitely many others. A similar argument applies to each monogenic inverse semigroup.

An inverse semigroup $S$ is pseudo-archimedean if no idempotent of $S$ is strictly below every idempotent of a free monogenic or bicyclic inverse subsemigroup. Clearly, by (1) of the proposition, every shortly linked inverse semigroup is pseudoarchimedean. If an idempotent is below infinitely many idempotents of a bicyclic subsemigroup then it must be below all of them. However, this is not obviously so for the free monogenic inverse subsemigroups and we now quote an example to show that the pseudo-archimedean property is strictly weaker than that of being shortly linked. 
EXAmple 3.2. [3, Example 3.11]. There is an aperiodic, E-unitary, pseudoarchimedean inverse semigroup that is not shortly linked. The semigroup is generated as an ideal by a single nonidempotent.

We may weaken the pseudo-archimedean hypothesis still further. Let us call $S$ faintly archimedean if whenever an idempotent $e$ of $S$ is strictly below every idempotent of a bicyclic or free monogenic inverse subsemigroup $\langle a\rangle$, then $e<a$. Adjoining a zero to a free monogenic inverse semigroup yields a faintly archimedean inverse semigroup that is not pseudo-archimedean. (K. H. Cheong [1] called $S$ weakly archimedean if this implication is required to hold for all monogenic inverse subsemigroups $\langle a\rangle$. She showed that any inverse semigroup whose lattice of convex inverse subsemigroups is lower semimodular has this property. The term 'archimedean' alludes to its use in earlier work by the author [6].)

Note that if a monogenic inverse subsemigroup $\langle a\rangle$ is of type $\left(k, \infty^{+}\right)$for some positive integer $k$, then $a^{k+1} a^{-k}$ generates its bicyclic kernel, where $a^{k+1} a^{-k} \leq a$. Thus $S$ is faintly archimedean if and only if whenever $e$ is below every idempotent of $\langle a\rangle$, where $E_{\langle a\rangle}$ is infinite, then $e<a$.

While this property will be an adequate hypothesis in the aperiodic case, it needs to be strengthened slightly to cover the general situation. Let us call $S$ quasi-archimedean if whenever an idempotent $e$ is (not necessarily strictly) below every idempotent of $\langle a\rangle$, where $a \in N_{S}$, then $e<a$.

Proposition 3.3. The following are equivalent for an inverse semigroup $S$ :

(1) $S$ is quasi-archimedean;

(2) if $a \in N_{S}, b<a$ and $b b^{-1}$ is below every idempotent of $\langle a\rangle$, then $b \in E_{S}$;

(3) $S$ is faintly archimedean and $\langle a\rangle$ is aperiodic for each $a \in N_{S}$;

(4) for each $a \in N_{S},\langle a\rangle$ is aperiodic and whenever $b<a, b b^{-1}<a^{n} a^{-n}$ and $b^{-1} b<$ $a^{-n} a^{n}$ for every positive integer $n$, then $b \in E_{S}$.

Proof. Throughout the proof, $e$ denotes an idempotent of $S$ and $a$ an element of $N_{S}$.

(1) $\Rightarrow$ (2) With $a, b$ as stated, then by (1), $b b^{-1}<a$. Thus $b=b b^{-1} a=b b^{-1}$.

(2) $\Rightarrow$ (3) If $e$ is strictly below each idempotent of $\langle a\rangle$ then $b=e a<a$ and $b b^{-1}=e$. So by $(2), b b^{-1}=b<a$. In particular, this holds whenever $\langle a\rangle$ is bicyclic or free. Now if $\langle a\rangle$ has no least idempotent then from the discussion in Section 1 it is aperiodic. In the alternative case it has a group kernel with identity $e$, say, which is clearly below every idempotent of $\langle a\rangle$ and so is below $a$. Again setting $b=e a$, by a similar argument to the above we obtain $b \in E_{S}$. But the kernel is generated by $b$ and so is trivial, that is, $\langle a\rangle$ is aperiodic.

(3) $\Rightarrow$ (1) Suppose $e$ is below every idempotent of $\langle a\rangle$. On the one hand, if $E_{\langle a\rangle}$ is infinite then, by hypothesis, $e<a$. On the other hand, if $E_{\langle a\rangle}$ is finite, then $\langle a\rangle$ is aperiodic and its least idempotent is $a^{n+1}=a^{n}$ for some positive integer $n$. Then $e \leq a^{n}=a^{n} a<a$.

(2) $\Rightarrow$ (4) Under the assumption of (4), $b b^{-1}=b\left(b^{-1} b\right) b^{-1} \leq a\left(a^{-n} a^{n}\right) a^{-1} \leq$ $a^{-(n-1)} a^{n-1}$ for $n>1$, so in fact $b b^{-1}$ is below every idempotent of $\langle a\rangle$ and (2) applies. Aperiodicity follows as in (2) $\Rightarrow(3)$.

(4) $\Rightarrow$ (3) Suppose $e$ is below every idempotent of $\langle a\rangle$, where $\langle a\rangle$ is bicyclic or free. Let $b=e a$. Then $b<a, b b^{-1}=e<a^{n} a^{-n}$ and $b^{-1} b=a^{-1} e a<a^{-1} a^{-n} a^{n} a$ for every positive integer $n$. By (4), ea $\in E_{S}$, that is, $e=e a<a$. 
COROLlary 3.4. An aperiodic inverse semigroup $S$ is quasi-archimedean if and only if it is faintly archimedean.

4. $\mathcal{L}$-determinability. In this section we generalize the result of Goberstein, cited earlier, on aperiodic inverse semigroups, and prove a similar result on completely semisimple nonaperiodic inverse semigroups.

LEMMA 4.1. Let $S$ and $T$ be inverse semigroups and suppose $\phi: S \rightarrow T$ is a bijection that induces an isomorphism $\mathcal{L} \mathcal{F}(S) \rightarrow \mathcal{L} \mathcal{F}(T)$, restricts to an isomorphism on $E_{S}$ and satisfies $\left\{a a^{-1}, a^{-1} a\right\} \phi=\left\{a \phi(a \phi)^{-1},(a \phi)^{-1} a \phi\right\}$ for every $a \in S$. If $a \in S, e \in E_{S}$ and $e<a$, then $e \phi<a \phi$.

Proof. Since the conclusion is unaffected by interchanging $a \phi$ with $a^{-1} \phi$, we may assume that $\phi$ preserves $\mathcal{L}$ and $\mathcal{R}$. Now $e<a a^{-1}$, so that since $\phi$ restricts to an isomorphism on $E_{S}$, e $\phi<\left(a a^{-1}\right) \phi=(a \phi)(a \phi)^{-1}$. Thus $e \phi \mathcal{R} e \phi a \phi$ and so if we put $c=(e \phi a \phi) \phi^{-1}$, then $c \mathcal{R} e$. Hence $e \phi a \phi \in E_{T}$, that is, $e \phi<a \phi$. For otherwise, applying Lemma 2.1 to $\phi^{-1}$ yields $c \leq a^{k}$ for some nonzero integer $k$, so that $c=e a^{k}=e$, a contradiction.

LEMMA 4.2. Let $S$ be any quasi-archimedean inverse semigroup and suppose that $\phi$ is a bijection of $S$ upon an inverse semigroup $T$ that (i) restricts to an isomorphism on $E_{S}$, (ii) preserves $\mathcal{L}$ and $\mathcal{R}$, (iii) restricts to an isomorphism on $\langle a\rangle$ for each $a \in N_{S}$ and (iv) induces an isomorphism of $\mathcal{L} \mathcal{F}(S)$ upon $\mathcal{L} \mathcal{F}(T)$. Then whenever $b<a$ in $S$ and $a \in E_{S} \cup N_{S}, b \phi<a \phi$.

Proof. Since $\phi$ restricts to an isomorphism on $E_{S}$, the conclusion holds if $a \in E_{S}$, so assume $a \in N_{S}$. If $b \in E_{S}$ then Lemma 4.1 applies. Otherwise, according to Lemma 3.3(4), either there is a greatest positive integer $n$ such that $b b^{-1}<a^{n} a^{-n}$ or the (left-right) dual of that statement holds. In the latter case, a dual argument applies, so consider the former possibility. Note that in conjunction with $b<a$, the stated inequality is equivalent to $b<a^{n} a^{-(n-1)}$. By Lemma 2.1, $b \phi \leq\left(a^{n} a^{-(n-1)}\right)^{k} \phi$ for some nonzero integer $k$.

Suppose first that $k=1$. Since $\phi$ restricts to an isomorphism on $\langle a\rangle,\left(a^{n} a^{-(n-1)}\right) \phi=$ $(a \phi)^{n}(a \phi)^{-(n-1)} \leq a \phi$, yielding the desired conclusion.

Next suppose $k>1$. Then by a simple inductive argument, $\left(a^{n} a^{-(n-1)}\right)^{k}=$ $a^{n+k-1} a^{-(n-1)}$. Hence $\left(b b^{-1}\right) \phi \leq\left(\left(a^{n+k-1} a^{-(n-1)}\right)\left(a^{n+k-1} a^{-(n-1)}\right)^{-1}\right) \phi=\left(a^{n+k-1} a^{-(n+k-1)}\right) \phi$. Now $\phi$ is an isomorphism on $E_{S}$, so $b b^{-1} \leq a^{n+k-1} a^{-(n+k-1)}$. Since, for $k>1$, $a^{n+k-1} a^{-(n+k-1)} \leq a^{n+1} a^{-(n+1)}$, the maximality assumption on $n$ yields $b b^{-1}=$ $a^{n+1} a^{-(n+1)}$. From $b<a$ we then obtain $b=a^{n+1} a^{-n}$ and so $b \phi=(a \phi)^{n+1}(a \phi)^{-n} \leq a \phi$, as required.

Finally, suppose $k<0$. Now, by similar arguments, $b^{-1} b \leq a^{n-k-1} a^{-(n-k-1)} \leq$ $a^{n} a^{-n}$ whence, since $b<a, b b^{-1} \leq a^{n+1} a^{-(n+1)}$ once more and the concluding argument of the preceding case applies.

\subsection{Aperiodic inverse semigroups.}

THEOREM 4.3. Let $S$ be any aperiodic, quasi-archimedean (equivalently, faintly archimedean) inverse semigroup and let $\Phi$ be an $\mathcal{L}$-isomorphism from $S$ to an inverse semigroup $T$ such that the weak isomorphism $\phi_{E}: E_{S} \rightarrow E_{T}$ is actually an isomorphism. Then $\Phi$ is induced by a unique isomorphism, namely the base bijection $\phi$ defined in Section 1 . 
Proof. Let $\phi: S \rightarrow T$ be as stated. According to [9, Theorem 3.1.5], if a mapping between two inverse semigroups is order preserving, restricts to a homomorphism on the semilattice of idempotents and preserves restricted products, then it is a homomorphism.

Let $a b$ be a restricted product. Then since $\phi$ respects Green's relations, $a \phi b \phi$ is also such a product and the resulting element is $\mathcal{H}$-related to $(a b) \phi$, whence equal, by aperiodicity.

Since $\phi$ induces $\Phi$, it induces its restriction $\mathcal{L F}(S) \rightarrow \mathcal{L} \mathcal{F}(T)$. By Lemma 4.2, it is order preserving. Hence it is an isomorphism.

In [4, Proposition 9], Goberstein constructed nonisomorphic aperiodic, completely semisimple inverse semigroups $S$ and $T$ whose partial automorphism semigroups, and hence their lattices of inverse subsemigroups, are isomorphic. It follows from his construction that the induced bijection restricts to an isomorphism between their semilattices of idempotents.

As discussed in the introduction, the restriction that the weak isomorphism $\phi_{E}$ : $E_{S} \rightarrow E_{T}$ be an isomorphism is not a strong one. As remarked there, if $S$ is $E$-unitary, then $\phi_{E}$ is an isomorphism on the idempotents of any ideal generated by a nontrivial $\mathcal{J}$-class. We may apply this fact to the semigroup in Example 3.2, which is $E$-unitary and is generated as an ideal by a single nonidempotent. Recalling that every pseudoarchimedean inverse semigroup is faintly archimedean, we may apply Theorem 4.3.

Other applications, some of which were noted by Goberstein, are to new proofs of determinability of free inverse semigroups and of simple inverse semigroups whose lattices of full inverse subsemigroups are modular.

4.2. Nonaperiodic, completely semisimple inverse semigroups. For Brandt semigroups with exactly two nonzero idempotents it is apparently unknown whether the base bijection $\theta$ is always an isomorphism (see [13, Problem 7.11]). However, Ershova proved the remarkable result [12, Theorem 44.6] (see also [13, Corollary 2.7]) that for Brandt semigroups with at least three nonzero idempotents, $\theta$ is always an isomorphism. In a similar fashion to the proof of the above lemma, this result may be incorporated into a result for completely semisimple inverse semigroups by noting that since $\theta$ preserves $\mathcal{L}$ and $\mathcal{R}$, if $a b=0$ in a Brandt semigroup then $a \theta b \theta=0$ in the image semigroup. The products that are nonzero are then precisely the restricted ones (those for which $a^{-1} a=b b^{-1}$ ) and the proof that $(a b) \theta=a \theta b \theta$ for such products in Brandt semigroups can simply be interpreted in the appropriate completely semisimple inverse semigroups, as follows. (Another proof may be obtained by inducing a lattice isomorphism between the corresponding principal factors of $S$ and $T$.)

LEMMA 4.4. Let $S$ be a completely semisimple inverse semigroup with the property that each nonaperiodic $\mathcal{D}$-class contains at least three idempotents. If $\Phi$ is an $\mathcal{L}$ isomorphism from $S$ to an inverse semigroup $T$ then the bijection $\theta: S \rightarrow T$ preserves restricted products.

THEOREM 4.5. Let $S$ be any completely semisimple, quasi-archimedean inverse semigroup in which each nonaperiodic $\mathcal{D}$-class contains at least three idempotents, and let $\Phi$ be any $\mathcal{L}$-isomorphism from $S$ to an inverse semigroup $T$ such that the weak isomorphism $\phi_{E}: E_{S} \rightarrow E_{T}$ is actually an isomorphism. Then $\Phi$ is induced by a unique isomorphism, namely the base bijection $\theta$ defined in Section 1. 
Proof. By Lemma 4.4, $\theta$ preserves restricted products and since it extends $\phi$, it restricts to an isomorphism on $E_{S}$. According to the first paragraph of the proof of Theorem 4.3, to prove it is an isomorphism it remains to show it is order preserving.

By Lemma 2.4, $\theta$ induces $\Phi$ and so induces an isomorphism $\mathcal{L F}(S) \rightarrow \mathcal{L F}(T)$; and by Proposition 3.3, $\langle a\rangle$ is aperiodic for each $a \in N_{S}$, so the restriction of $\theta$ agrees with $\phi$ thereon and is therefore an isomorphism. Hence, if $b<a$ in $S$ and $a \in E_{S} \cup N_{S}$, then $b \phi<a \phi$, by Lemma 4.2.

To conclude the proof, suppose $b<a$, with $a \notin E_{S} \cup N_{S}, a \in H_{f}, f \in E_{S}$. Put $e=b b^{-1}<a a^{-1}=f$. Since $D_{a}$ is nonaperiodic, $R_{f}$ contains an element $r$ of $N_{S}$ and $a=r r^{-1} a=r\left(a^{-1} r\right)^{-1}$, where $a^{-1} r \in H_{r} \subset N_{S}$ (similarly to the definition of $\theta$ in $\S 1$ ). Then $b=e b=e f b=e r r^{-1} b=(e r)\left(b^{-1} r\right)^{-1}$, where this last product is easily verified to be restricted. According to the first sentence of the proof, $b \theta=(e r) \theta\left(b^{-1} r\right)^{-1} \theta=$ (er) $\theta\left(\left(b^{-1} r\right) \theta\right)^{-1}$. But $e r<r \in N_{S}$ and $b^{-1} r<a^{-1} r \in N_{S}$, so applying the previous paragraph we obtain $(e r) \theta<r \theta$ and $\left(b^{-1} r\right) \theta<\left(a^{-1} r\right) \theta$, whence $\left(\left(b^{-1} r\right) \theta\right)^{-1}<\left(\left(a^{-1} r\right) \theta\right)^{-1}$. Hence $b \theta<r \theta\left(\left(a^{-1} r\right) \theta\right)^{-1}=a \theta$ (reversing the previous argument), as required.

We now give an example to show that the quasi-archimedean hypothesis is necessary for the above theorem to hold. We first provide a general construction of certain $\mathcal{L}$-isomorphic semigroups.

Proposition 4.6. Let $B$ be an inverse semigroup and $A$ an inverse semigroup with zero. Let $S, S^{\prime}$ be retract extensions of $B$ by $A$, via partial homomorphisms $\psi, \psi^{\prime}$ that satisfy $\langle a \psi\rangle=\left\langle a \psi^{\prime}\right\rangle$ for all $a \in A^{*}$. Then the identity map $I: \mathcal{L}(S) \rightarrow \mathcal{L}\left(S^{\prime}\right)$ is an isomorphism.

Proof. Let $U \in \mathcal{L}(S)$. Let $U^{\prime}$ denote the set $U$, considered as an inverse subsemigroup of $S^{\prime}$. In both $S$ and $S^{\prime}, A^{*}$ and $B$ are closed under the inverse operation and under restricted products, since each is a union of $\mathcal{D}$-classes. By hypothesis, $\psi$ and $\psi^{\prime}$ agree on the idempotents of $A^{*}$, so $E_{U^{\prime}} \cong E_{U}$. Finally, let $u, v \in S^{\prime}$, with $v v^{-1}, u \in U^{\prime}$. If both $u, v$ belong to either $B$ or $A^{*}$ then since the operations on $U, U^{\prime}$ coincide there, $v \in U^{\prime}$. The remaining case is where $v \in B$ and $u \in A^{*}$. Now $v<u$ in $S^{\prime}$ if and only if $v \leq u \psi^{\prime}$ in $B$. Since $\left\langle u \psi^{\prime}\right\rangle=\langle u \psi\rangle, u \psi^{\prime} \leq(u \psi)^{n}$ for some (without loss of generality) nonzero integer $n$. Now if $u^{n} \in A^{*}$ then $(u \psi)^{n}=u^{n} \psi$; if not, $(u \psi)^{n}=u^{n}$. In either event, $v \leq u^{n} \in U$ in $S$ and so $u \in U$ in $S$, that is, $u \in U^{\prime}$ in $S^{\prime}$. By Lemma 2.2, $U^{\prime} \in \mathcal{L}\left(S^{\prime}\right)$.

By symmetry, this map yields an order isomorphism between $\mathcal{L}(S)$ and $\mathcal{L}\left(S^{\prime}\right)$.

Now let $A=\left\langle a: a^{5}=a^{2}\right\rangle$ and let $B$ be the Brandt semigroup $\mathcal{M}^{0}(X, G, X, I)$, where $G=\left\langle g: g^{3}=1\right\rangle$ and $X=\{1,2,3\}$ (see [11] for this construction). Mapping $a$ to $g$ induces a partial homomorphism $\psi: A^{*} \rightarrow G$; a partial homomorphism $\psi^{\prime}$ may be defined similarly by mapping $a$ to $g^{2}$. Here $\langle a \psi\rangle=G=\left\langle a \psi^{\prime}\right\rangle$. Since $G$ is isomorphic to the maximal subgroup $H_{(1,1,1)}$ of $B$, we may regard $\psi$ and $\psi^{\prime}$ as mapping into $B$. The hypotheses of the proposition having been met, the identity map is an $\mathcal{L}$-isomorphism between the two semigroups $S, S^{\prime}$ defined there. Since the induced partial bijection $\phi$ is just the identity map it follows from the construction of $\theta$ (and the fact that the copies of $B$ in $S$ and in $S^{\prime}$ are isomorphic, by construction) that $\theta$ is also the identity map. However the identity map between $S$ and $S^{\prime}$ is not an isomorphism, since $a^{2}=(a \psi)^{2}=g^{2}$ in $S$ whereas $a^{2}=\left(a \psi^{\prime}\right)^{2}=g^{4}=g$ in $S^{\prime}$. As noted above, if $\Phi$ is induced by any isomorphism, that mapping must be $\theta$. Hence we have exhibited the following.

EXAMPLE 4.7. There exist two finite inverse semigroups, in each of which every nonaperiodic $\mathcal{D}$-class possesses at least three idempotents, and an $\mathcal{L}$-isomorphism between them that induces an isomorphism $\phi_{E}$ between their semilattices of 
idempotents and is induced by a bijection (namely $\theta$ ) that preserves restricted products, but which is not induced by any isomorphism between the semigroups themselves. (In particular, the base bijection $\theta$ is not an isomorphism).

\section{REFERENCES}

1. K. H. Cheong, Closed inverse subsemigroup lattices of inverse semigroups, Ph.D. Dissertation (Marquette University, 1994).

2. K. H. Cheong and Peter R. Jones, Inverse semigroups determined by their lattices of convex inverse subsemigroups, I, Algebra Universalis 49 (2003), 51-80.

3. K. H. Cheong and Peter R. Jones, Inverse semigroups determined by their lattices of convex inverse subsemigroups, II, Algebra Universalis 49 (2003), 81-106.

4. Simon M. Goberstein, Inverse semigroups with isomorphic partial automorphism semigroups, J. Austral. Math. Soc. Ser. A 47 (1989), 399-417.

5. S. M. Goberstein, A note on shortly connected inverse semigroups, Bull. Austral. Math. Soc. 43 (1991), 463-466. 466.

6. P. R. Jones, Distributive inverse semigroups, J. London Math. Soc. (2) 17 (1978), 457-

7. P. R. Jones, Inverse semigroups determined by their lattices of inverse subsemigroups, J. Austral. Math. Soc. Ser. A 30 (1981), 321-346.

8. Peter R. Jones, Inverse semigroups and their lattices of inverse subsemigroups, in Lattices, semigroups and universal algebra, ed. J. Almeida et al. (Plenum Press, New York, 1990).

9. Mark V. Lawson, Inverse semigroups (World Scientific Publ. Co., Singapore, 1998).

10. W. D. Munn, Free inverse semigroups, Proc. London Math. Soc. (3) 29 (1974), 385-404.

11. M. Petrich, Inverse semigroups (Wiley, New York, 1984).

12. L. N. Shevrin and A. J. Ovsyannikov, Semigroups and their subsemigroup lattices (Kluwer, Dordrecht, 1996).

13. A. J. Ovsyannikov, Lattice isomorphisms of completely 0 -simple inverse semigroups, Semigroup Forum 62 (2001), 115-145. 